EUROPEAN LABORATORY FOR PARTICLE PHYSICS

CERN-PPE/97-19

6 January 1997

\author{
DEVELOPMENT OF HIGH RATE MSGCS: \\ OVERVIEW OF RESULTS FROM RD-28 \\ Fabio Sauli \\ CERN, CH-1211 Geneva, Switzerland
}

\begin{abstract}
Many laboratories world-wide have contributed to the R\&D project RD-28 at CERN (development of high rate micro-strip gas chambers). Various aspects of the design and use of the detector have been studied, in particular those connected with long-term operation in a high radiation flux. This paper summarizes some major outcomes of the research: the development of controlled resistivity substrates, the studies of pollution-induced ageing processes, the effects of substrate and metallization on performance, the operating characteristics in beam conditions.
\end{abstract}

Invited paper at the 5th International Conference on Advanced Technology and Particle Physics Como, Italy, 7-11 October 1996 


\section{Development of high rate MSGCs: overview of results from RD-28}

Fabio Sauli

CERN, CH-1211 Geneva, Switzerland

Many laboratories world-wide have contributed to the R\&D project RD-28 at CERN (development of high rate micro-strip gas chambers). Various aspects of the design and use of the detector have been studied, in particular those connected with long-term operation in a high radiation flux. This paper summarizes some major outcomes of the research: the development of controlled resistivity substrates, the studies of pollution-induced ageing processes, the effects of substrate and metallization on performance, the operating characteristics in beam conditions.

\section{INTRODUCTION}

Micro-Strip Gas Chambers (MSGC [1]) have attracted a large interest due to promising performances: very good position and track resolution, high rate capability. Medium and longterm stability problems have been met however at high rates due to substrate charging up, and ageing due to gas polymerization. To coordinate the research on MSGC by various groups, many of them working on tracking devices for the LHC experiments, an R\&D program was started at CERN as RD-28; several reports relate the progress of the project [2-4]. Two workshops on Micro-strip Chambers have also been organized, hosted by INFN-Legnaro and IN2P3-Lyon; the proceedings include a large number of contributions on development and applications of the new detectors $[5,6]$.

\section{RD-28 RESEARCH PROGRAM}

The RD-28 research program covered several topics related to generic research and development on MSGCs, to requirements of tracking for LHC experiments, and to applications in other fields. This paper will address only some major issues:

- development of substrates for MSGC manufacturing, with characteristics apt at eliminating gain variations due to polarization and charging up at high rates;

- choice of operating gases and pollution- free assembly materials, to obtain acceptable lifetimes under sustained irradiation;

- test-beam measurements;

- special, application-specific detectors.

\section{THE MICRO-STRIP GAS CHAMBER}

The micro-strip gas chamber (Fig. 1) consists of alternating thin metal strips, anodes and cathodes, typically 10 and $100 \mu \mathrm{m}$ wide, on an insulating support at a pitch of a few hundred microns. An upper drift electrode delimits the sensitive gas volume where electrons are released by ionizing radiation. The back side can also have a fielddefining electrode: with thin substrates, it can be segmented for two-dimensional localization. Applying proper potentials to the electrodes, an electric field builds up such that electrons released in the drift space are collected and multiplied reaching the anodes.

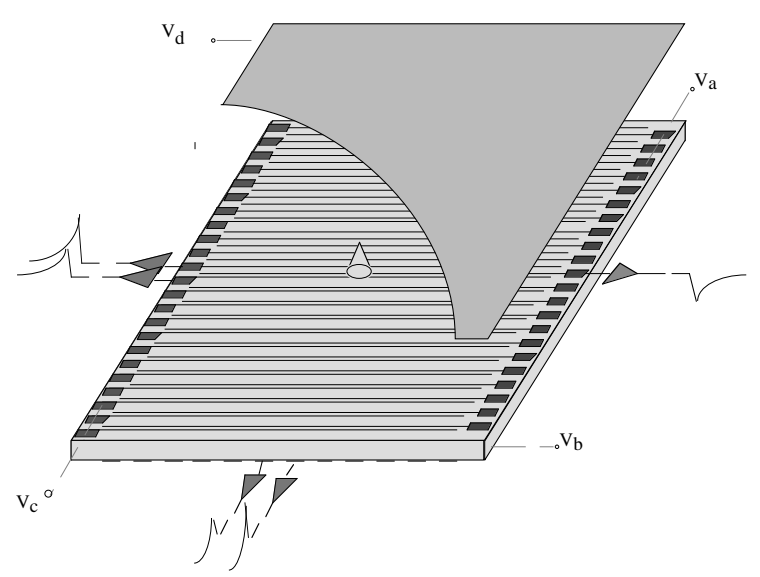

Fig. 1: Schematics of a two-dimensional MSGC.

MSGCs have very promising features: - proportional gains over $10^{4}$ [7];

- position accuracy around $30 \mu \mathrm{m} \mathrm{rms} \mathrm{[8];}$

- rate capability above $10^{6} \mathrm{~mm}^{-2} \mathrm{~s}^{-1}$ [9];

- energy resolution $\sim 11 \%$ fwhm for $5.9 \mathrm{keV}$ X-rays [10];

- moderate costs, typically around $200 \$$ for an active substrate area $10 \times 10 \mathrm{~cm}^{2}$. 
Some stability problems have however been met: gain modifications due to substrate polarization and charging up, and permanent deterioration (ageing) during sustained irradiation [9, 11]. The physical parameters used to manufacture and operate the detectors (substrate material, metal of strips, gas mixture and purity) play a dominant role in determining the long-term stability of operation of the devices; the situation is complicated by the interdependence of the various parameters. A big effort has been undertaken, within and outside RD28, to understand all aspects of MSGC operation, to improve their performances and lifetime and to [11-13] and during irradiation [13-15]. These effects are attributed to the modification of the electric field by substrate polarization following the application of the potentials, to charging up of the insulator by electrons and ions produced in the avalanches, and to ions' migration within the substrate. Examples are shown in Figs. 2 and 3. While for moderate radiation fluxes high resistivity supports can and have been adopted (for example for the HERMES experiment [16]), this can be a source of serious operating instabilities at higher rates.

Use of a substrate with lower resistivity and electronic conductivity eliminates the polarization and surface charging processes up to very high rates; it results also in more stable operation and reduced ageing rates.

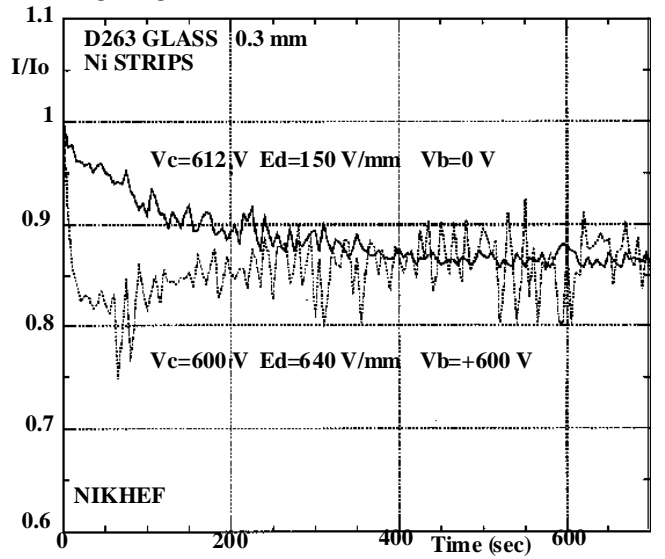

Fig. 2: Initial stabilization of gain of a MSGC made on boro-silicate glass reduce the manufacturing costs, an essential goal in view of use in LHC trackers.

\section{CHOICE OF THE SUBSTRATE}

Proper choice of the substrate material is crucial to obtain stable operation of the detector. Most commercially available insulators with good surface quality have very high resistivity, above $10^{16} \Omega \mathrm{cm}$, and ionic conductivity; it has been found that this results in operating instabilities of the MSGCs immediately after power on

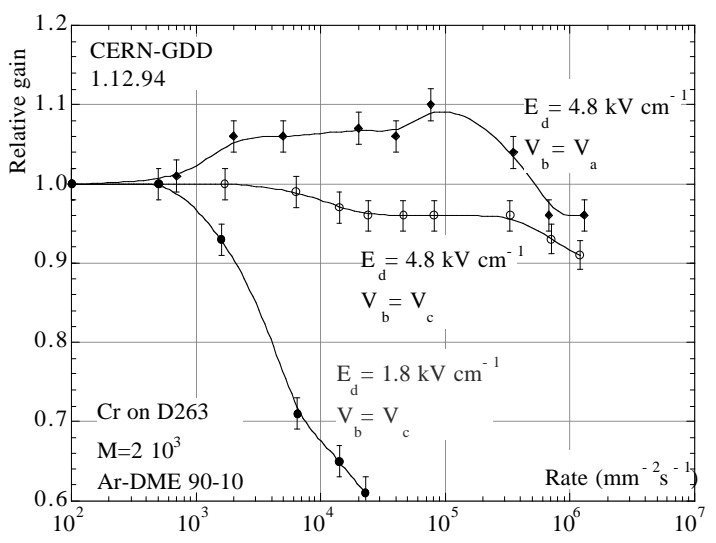

Fig. 3: Rate dependence of gain for a MSGC made on D-263 glass, for several conditions.

The best results have been obtained with electron-conducting glass having resistivity in the range $10^{9}-10^{12} \Omega \mathrm{cm}[9,17,18]$ or surface modified supports in the range $10^{14}-10^{16} \Omega$ /square. Several methods of conditioning insulating supports to obtain the desired surface resistivity have been developed. Fig. 4 [19] shows the reduction in surface resistivity of Desag D-263 glass after implantation with $10^{15}$ boron ions $\mathrm{cm}^{-2}$; the gain stability under continuous irradiation is largely improved, see Fig. 5 [19].

Direct deposition by sputtering of an electronconducting lead silicate glass layer has been developed with good results in terms of uniformity and stability [20]. 


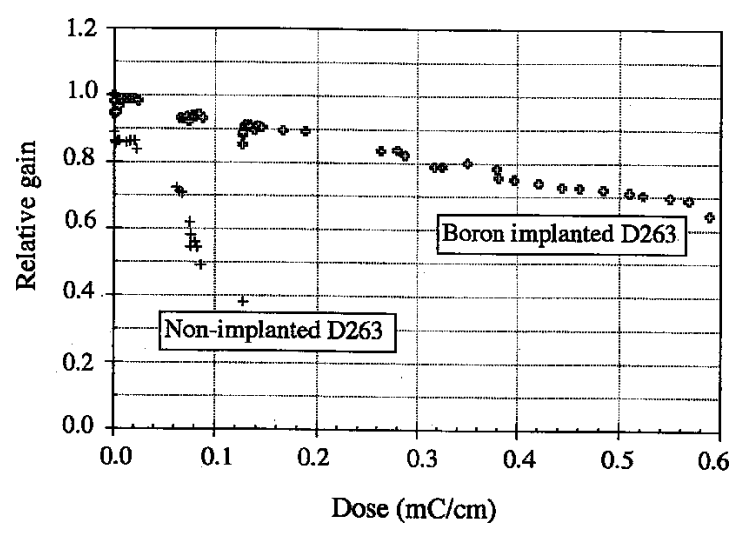

Fig. 4: Comparison of gain stability for standard and boron implanted glass.

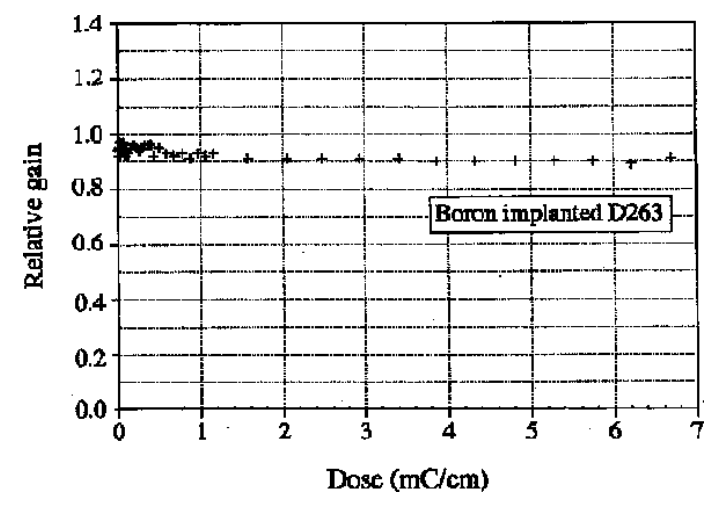

Fig. 5: Improved stability at high radiation doses of a MSGC made on implanted glass.

Due to the moderate dependence of gain on surface resistivity, MSGCs manufactured on sputtered glass exhibit an acceptable gain uniformity; the rate capability is largely enhanced (Fig. 6 [21]). The ageing behavior is also improved (see the next section); work is in progress to improve the manufacturing by using mobile sputtering targets.

Very good results have been obtained with thin diamond-like carbon (DLC) coatings obtained by Chemical Vapor Deposition (CVD [22]; DLC coated plates have been extensively tested and appear very stable in time. Fig. 7 shows the rate capability of a chamber exposed to high rate $x$-ray fluxes, with no gain drop up to $2.10^{6}$ counts $\mathrm{mm}^{-2} \mathrm{~s}^{-1}$ at an avalanche size of about $10^{5}$ electrons.

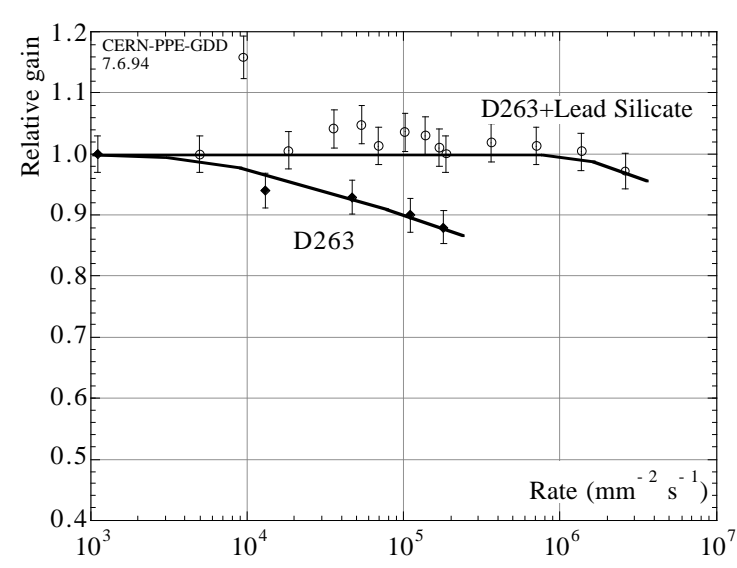

Fig. 6: Rate capability of an MSGC made on sputtered lead silicate glass.

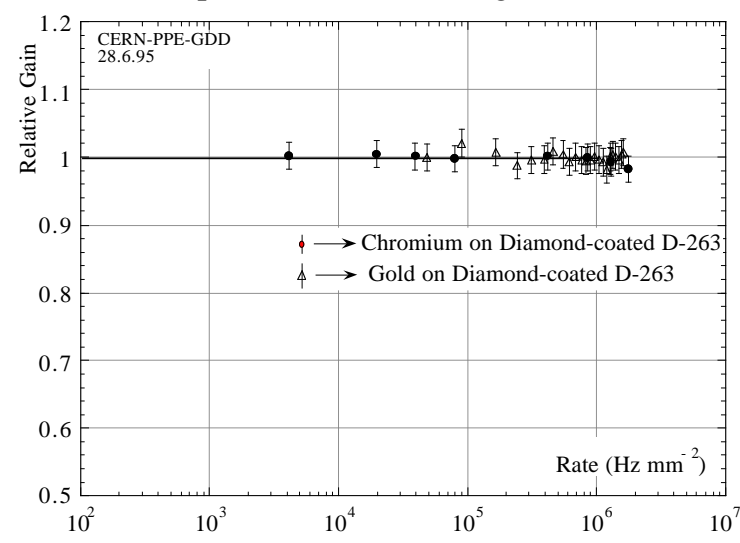

Fig. 7: Rate capability of MSGCs made on diamond-like coated glass.

Other technologies for surface resistivity control by thin-layer coating have been explored, such as ion-beam sputtering of semi-conducting glass, hydrogenated silicon, low pressure CVD of hydrogenated carbon [23]. A property of some layers is the variation of resistivity with the electric field; the effect of this behavior on the operation of the detectors remains to be investigated.

Over-coating, covering an existing MSGC structure with a thin resistive layer, has also been tried by several groups and is intrinsically simpler to implement since it avoids metal adhesion problems in manufacturing the chamber; the long-term stability of the layers, that can be damaged by the electron and ion currents is however still not satisfactory [24].

\section{MSGC OPERATION}


Operating parameters and features of MSGCs such as gas gain, space and energy resolution, signal characteristics, noise and discharge rate have been studied for various gas mixtures, gap, pitch, width of the electrodes, support thickness and potential of the back electrode; the best results in gain and stability of operation have been obtained using mixtures of argon or neon with dimethyl-ether (DME) $[19,25,26]$. Other gases used include mixtures with $\mathrm{CO}_{2}$ and $\mathrm{CF}_{4}$. Fig. 8 shows a set of gain curves measured with MSGC plates having identical geometry ( $7 \mu \mathrm{m}$ anodes and $200 \mu \mathrm{m}$ pitch) and different cathode strip widths; an optimum is reached for around $100 \mu \mathrm{m}$ [27].

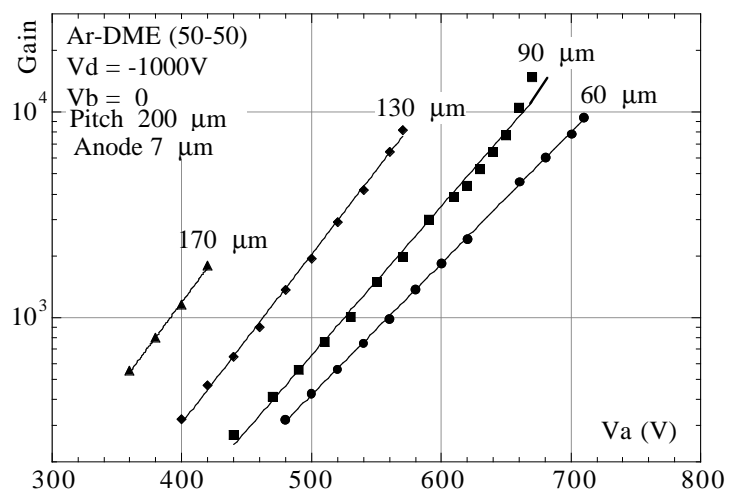

Fig. 8: Maximum gain reachable with MSGCs having different cathode widths.

One expects also a large variation in the maximum gain safely reached depending on the manufacturing technology (affecting the detailed shape of the edges) and perhaps the nature of the electrodes; indeed, maximum gains reached in practice vary from less than a thousand to more than $10^{4}$.

The effect of neutron irradiation on MSGC has been studied experimentally and theoretically, and the results suggest a considerable contribution to background noise; the choice of materials for MSGC manufacturing, of the strip's metal and of operating gas may play a critical role in the background levels, and are investigated. Recoil protons or activation gammas can easily release in the gas several hundred $\mathrm{keV}$, as against the few $\mathrm{keV}$ produced by minimum ionizing particles [28]. In presence of heavily ionizing tracks, the maximum safe operating gain is strongly reduced, as shown in
Fig. 9 [27]. The results of a systematic investigation on this point are discussed in another contribution to this conference [29].

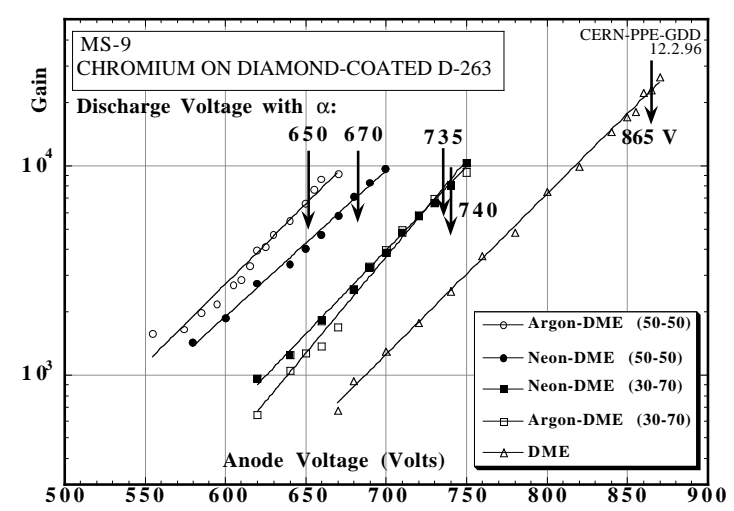

Fig. 9: Gain and maximum with alpha particles in several gas mixtures.

\section{AGEING}

Ageing, the fast degradation of the performance of the detectors during irradiation, is the most serious problem encountered with all gas detectors; MSGCs have been found to be particularly prone to ageing because of the small effective electrode area. Systematic investigations of ageing under sustained irradiation have been performed using MSGC plates manufactured on different substrates and in various operating conditions. In optimal laboratory conditions, a long-term survival without degradation up to a collected charge above $100 \mathrm{mC} \mathrm{cm}^{-1}$ has been demonstrated [9, 14, 26, 30-33]. An example of gain measured as a function of collected charge is provided in Fig. 10, obtained in a chamber with chromium strips on diamond-like coated glass, and using construction materials found in the outgassing studies [22, 33]. 


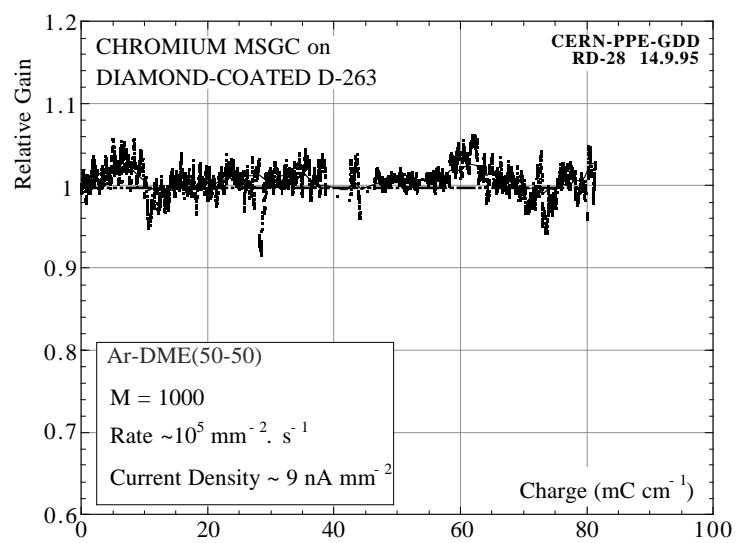

Fig. 10: Long-term irradiation of a chromium MSGC made on DLC coated glass.

Most ageing tests are accelerated exposing the detector to a high intensity X-ray flux, and taking as normalization factor the total collected charge per unit length of strips. It has been found however that use of excessive radiation rates (current densities) results in an over-optimistic estimate of the ageing rates [14]. While the exact mechanism of this behavior is not clear, the observation invalidates some measurements realized at excessive current densities and casts a general doubt on the adequacy of accelerated ageing tests.

Together with the purity of the gas system, important factors in determining ageing rate are the nature of the support and the metal used for the strips; the strength of the drift field also seems to play a role. A clear improvement has been observed replacing aluminum with copper, gold and nickel for the strips (see Fig. 11 [34]). Fig. 12 shows the large difference in ageing rate measured on two MSGCs manufactured in aluminum or gold on electron-conducting glass [26]; a similar result has been obtained with detectors made on boro-silicate glass sputtered with a thin layer of low resistivity S8900 glass [35].

Ageing of MSGCs is still a rather controversial issue, rich of contradictory results; it seems clear however that only a proper choice of materials and gases can guarantee a stable, long-term operation.

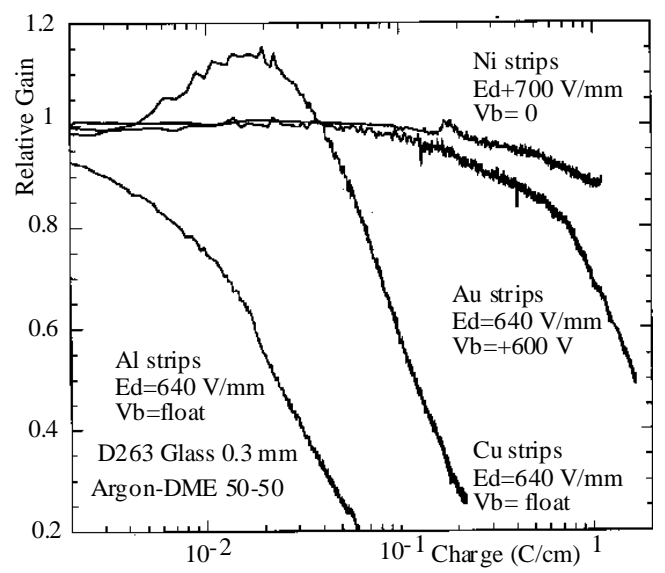

Fig. 11: Improvement in ageing rate of MSGCs made with $\mathrm{Al}, \mathrm{Cu}, \mathrm{Au}$ and Ni.

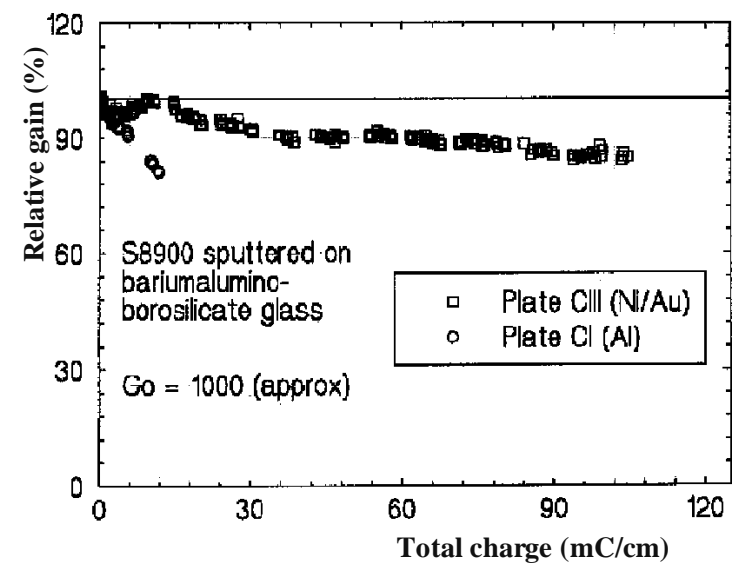

Fig. 12: Ageing of a chamber made on nude and S8900 coated D-263 glass.

\section{ASSEMBLIES, SPECIAL GEOMETRY}

Using the outcomes of the systematic research on materials, the CERN group has developed an "all clean" MSGC assembly that is simple and light enough to be considered a good approximation for a realistic module in the large detector arrays. As shown in Fig.13, a MSGC module is manufactured with a thin $(1 \mathrm{~mm})$ rectangular polymer frame glued with low outgassing epoxy directly on the MSGC plate; a second thin glass plate, conductive on the inner side, is glued to the frame and constitutes the drift electrode; holes in the frame allow to bring the gas in and out with thin stainless steel or aluminum tubes. A set of fully equipped detectors has been installed in a beam for a long-term performance 
study; the results are reported elsewhere in this conference [36].

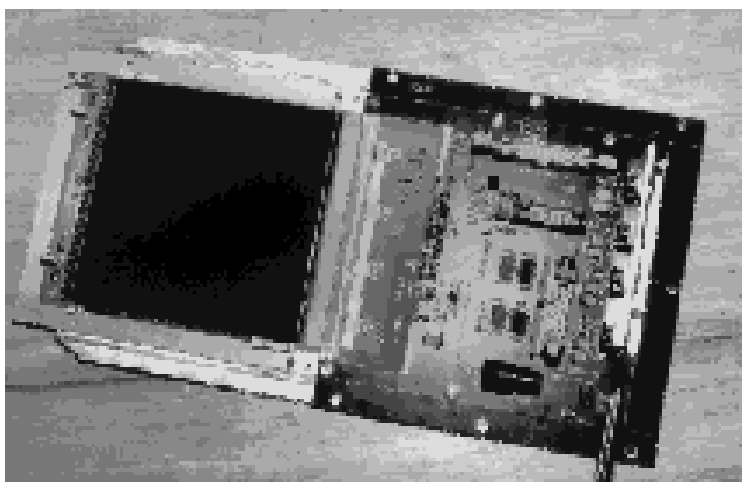

Fig. 13: A fully assembled MSGC with 100x100 $\mathrm{mm} 2$ active area and fast read-out.

Other assembly schemes have been developed within the collaboration, aimed at making realistic prototypes for the LHC experiments. The picture in Fig. 14 shows a "banana" shaped detector with two large wedge-shaped MSGC, developed for the CMS forward detector [37]; the readout electronics is included in the gas volume. Ageing characteristics of this "open geometry" design have been studied, with positive results [29].

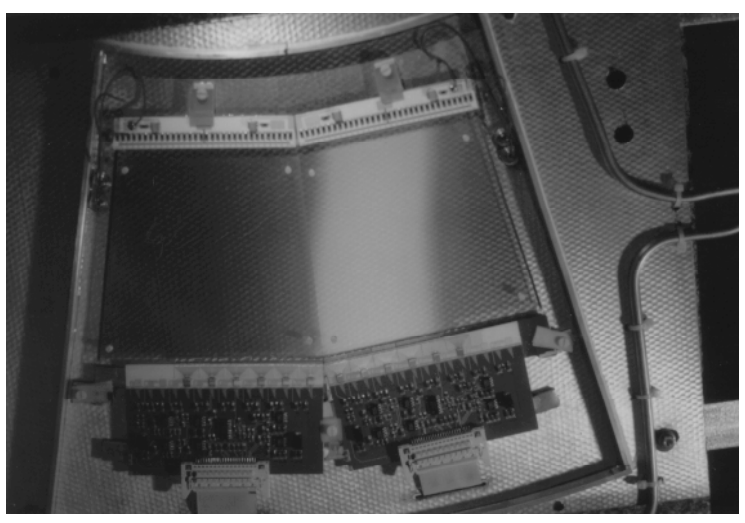

Fig. 14: Twin "banana" module developed for the Forward MSGC CMS detector.

\section{BEAM TESTS}

MSGCs set-ups have been successfully used in physics experiments (SMC [38], NA45 and HERMES [39]); smaller systems have been installed in test beams to measure the intrinsic properties of the devices and to gain experience with medium-term operation Efficiency and timing properties of MSGCs in detecting minimum ionizing particles have been studied in detail [40]. Fig. 15 shows the total charge signal distribution for perpendicular minimum ionizing particles and the corresponding noise spectrum; Fig.16 provides the detection efficiency and noise occupancy as a function of threshold [41]). Fig. 17 shows computed and measured values of the space accuracy as a function of pitch; the angular dependence of cluster size and efficiency has also been studied [40].

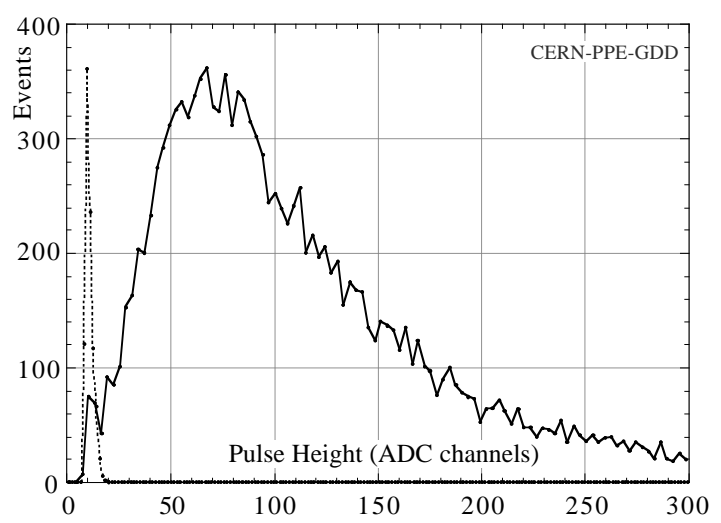

Fig. 15: Total charge for minimum ionizing particles and noise spectrum.

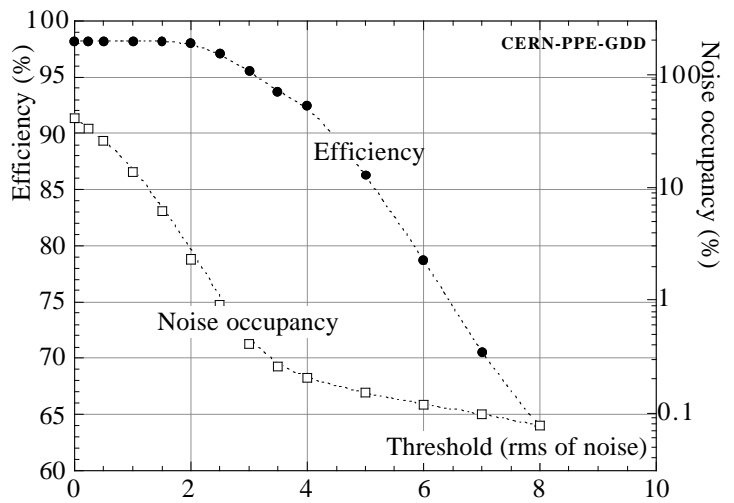

Fig. 16: Efficiency and noise occupancy as a function of electronics threshold. 


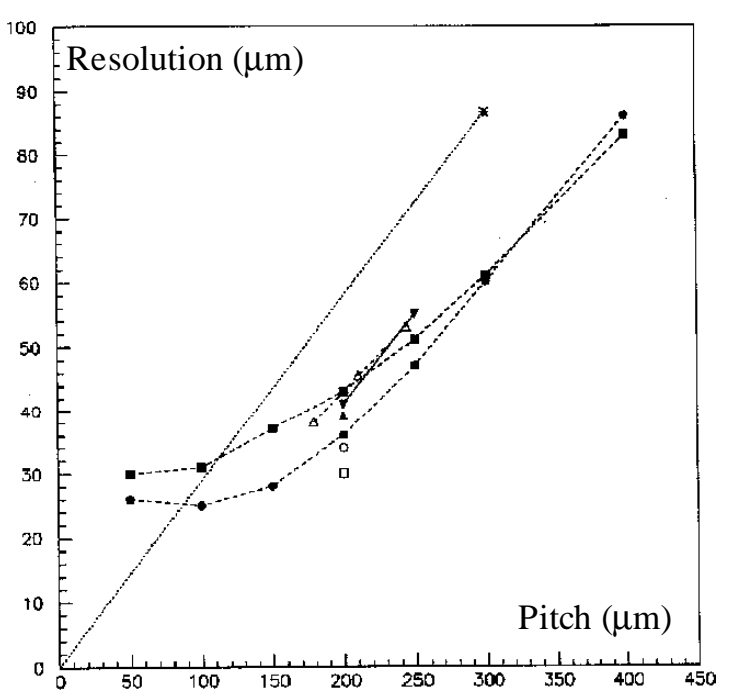

Fig. 17: Space resolution as a function of pitch.

Within the CMS tracker group, and as a continuation of the RD-28 development, a system of prototype MSGCs with an active area of $10 \times 10 \mathrm{~cm} 2$ and manufactured in different technologies (metals, coatings) has been operated continuously for almost one year in a high intensity muon beam $\left(10^{7}\right.$ particles per second over few $\mathrm{cm}^{2}$ ). Some results of this run are described in a separate paper submitted to this conference [36].

\section{SPECIAL DETECTORS}

Several groups have started interesting developments of special configurations or applications of the detectors A two-stage operation of the device at low pressures that allows to obtain very high gains has been demonstrated [42]; coupled to a CsI photo cathode the two-step low pressure MSGC is a very promising detector for UV light imaging (see Fig. 18).

Alternative geometries to the simple multiple strip patterns have been also developed. Shown in Fig. 19, the micro-dot chambers consists in a matrix of adjacent metal rings (the cathodes) with an anode "dot" in the centre [43, 44]. For convenience, of read-out, rows of dots are connected together; the device is however intrinsically capable of pixel readout. Gains well above $10^{4}$ have been demonstrated (see Fig. 20).

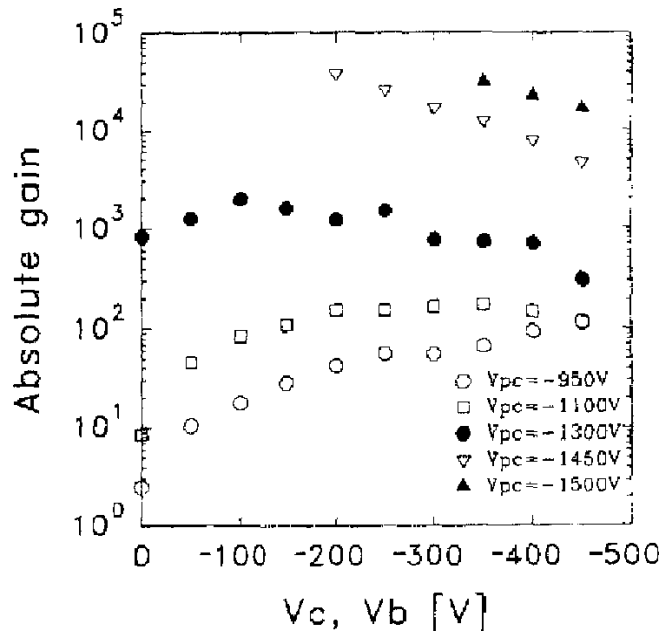

Fig. 18: Single electron detection in low-pressure MSGC with CsI photocathode.

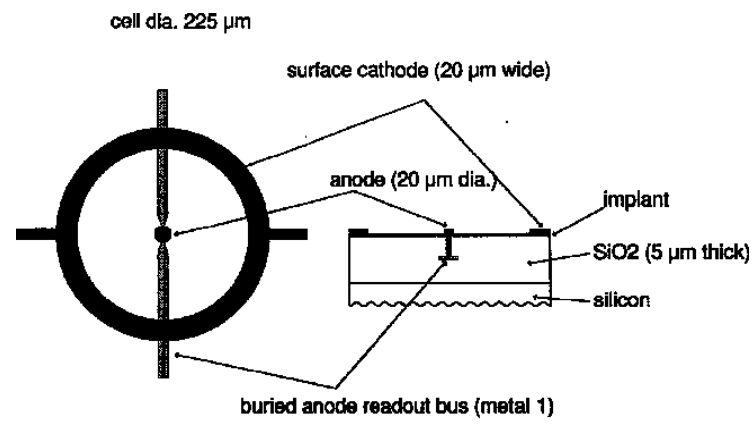

Fig. 19: Schematics of the microdot chamber.

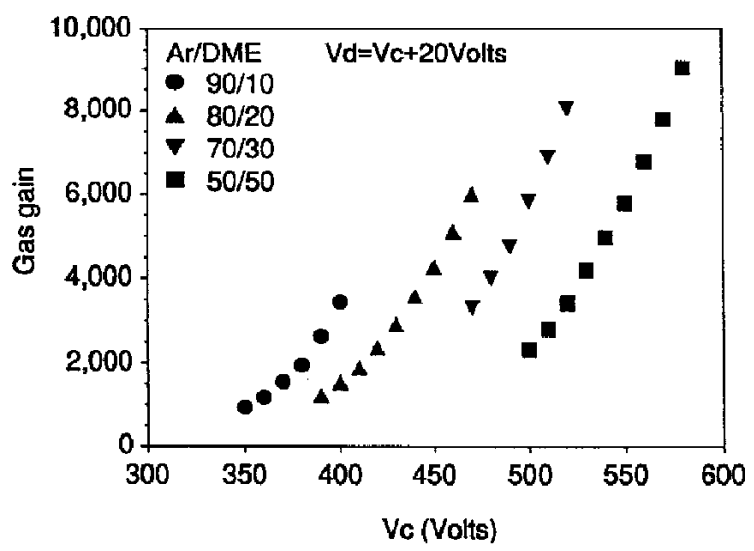

Fig. 20: Gain curves measured with a micro-dot chamber in several gas mixtures.

REFERENCES 
[1] A. Oed, Nucl. Instrum. Methods A263 (1988) 351.

[2] F. Sauli, CERN/DRDC/93-34 (1993).

[3] F. Sauli, CERN/DRDC/94-45 (1995).

[4] F. Sauli, CERN/LHCC 96-18 (1996).

[5] Proc. Int. Workshop on Micro-Strip Gas Chambers (Legnaro, Italy, 1995).

[6] Proc. Int. Workshop on Micro-Strip Gas Chambers (Lyon, 1996).

[7] T. Beckers et al., Nucl. Instrum. Methods A346 (1994) 95.

[8] F. Angelini et al., Nucl. Physics 23A (1991) 254.

[9] R. Bouclier et al., Nucl. Instrum. Methods A332 (1993) 100.

[10] F. Angelini et al., Proc. LHC Workshop (Aachen, 1990), 222.

[11] J.E. Bateman et al., RAL-92-085 (1992).

[12] R. Bouclier et al., Nucl. Instrum. Methods A323 (1992) 240.

[13] J.E. Bateman et al., RAL-95-038 (1995).

[14] R. Bouclier et al., Nucl. Instrum. Methods 367 (1995) 168.

[15] F.D. van den Berg et al., (1996).

[16] T. Henkes et al., Proc. Int. Workshop on Micro-Strip Gas Chambers (Lyon, 1996), 143.

[17] G.D. Minakov et al., Nucl. Instrum. Methods A326 (1993) 566.

[18] R. Bouclier et al., IEEE Trans. Nucl. Sci. NS41 (1994) 821.

[19] J.M. Brom et al., CRN 95-14 (1995).

[20] G. Della Mea et al., J. Am. Ceramic Soc. 76 (1993) 2930.

[21] R. Bouclier et al., Proc. Int. Conf. on MicroStrip Gas Chambers (Legnaro 1994), 39.

[22] R. Bouclier et al., Nucl. Instrum. Methods A 369 (1995) 328.

[23] A.V. Bagulya et al., Int. Workshop on MicroStrip Gas Chambers, Lyon ( 1995).

[24] A. Barr et al., Proc. 5th Int. Conf. on Advanced Technology and Particle Physics (Villa Olmo, 1996) .

[25] L. Alunni et al., Nucl. Instrum. Methods A348 (1994) 344.

[26] J.E. Bateman et al., RAL-94-114 (1994).

[27] R. Bouclier et al., Nucl. Instrum. Methods A365 (1995) 65.

[28] M.J. Esten et al., Proc. Int. Workshop on Micro-strip Gas Chambers (Lyon, 1995), 321.
[29] B. Boimska et al., Proc. 5th Int. Conf. on Advanced Technology and Particle Physics (Villa Olmo, 1996).

[30] M. Salomon et al., TRI-PP 94-24 (1994).

[31] M. Salomon et al., IEEE Trans. Nucl. Sci. NS41 (1994) 817.

[32] R. Bouclier et al., Nucl. Instrum. Methods A348 (1994) 109.

[33] R. Bouclier et al., Subm. Nucl. Instrum. Methods (1996) .

[34] F.D. van den Berg et al., Proc. Int. Workshop on Micro-Strip Gas Chambers (Lyon, 1995), 285.

[35] J.E. Bateman et al., RAL-TR-95-032 (1995).

[36] A. Barr et al., Proc. 5th Int. Conf. on Advanced Technology and Particle Physics (Villa Olmo, 1996).

[37] S. Barthe et al., Proc. Int. Workshop on MicroStrip Gas Chambers (Lyon, 1995), 107.

[38] M.H.J. Geijsberts et al., NIKHEF-H/94-12 (1994).

[39] T. Henkes et al., Proc. Int. Workshop on Micro-Strip Gas Chambers (Lyon, 1995), 143.

[40] S. Snow et al., Proc. Int. Workshop on MicroStrip Gas Chambers (Lyon, 1995), 127.

[41] R. Bouclier et al., Nucl. Instrum. Methods A367 (1995) 163.

[42] A. Breskin et al., Nucl. Instrum. Methods A345 (1994) 205.

[43] S.F. Biagi et al., Nucl. Instrum. Methods A366 (1995) 76.

[44] S. Biagi, Proc. 5th Int. Conf. on Advanced Technology and Particle Physsics (Villa Olmo, 1996). 